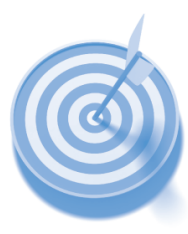

\author{
Katherine J Haxton* and \\ David J McGarvey \\ School of Physical and \\ Geographical Sciences \\ (Chemistry) \\ Keele University \\ Staffordshire \\ ST5 5BG
}

*k.j.haxton

@chem.keele.ac.uk

\section{Screencasting as a means of providing timely, general feedback on assessment}

\begin{abstract}
Feedback has been highlighted as a key area for improvement in teaching in higher education following recent National Student Survey results. While feedback may be provided in a variety of forms, both general and specific to individual students, there is often a need to provide general feedback to a whole class without consuming valuable and limited contact time.
\end{abstract}
Screencasting involves recording a short video clip of a computer screen with narration. It can be used to demonstrate various computer programmes, how to perform data analysis and to provide feedback on assessment, both individually and to the class as a whole. Camtasia or other screencast facilities were used to produce short videos of solutions to problem based assessments, incorporating general feedback and addressing misconceptions that had arisen. The resulting video files can be made available through virtual learning environments or on external websites giving the students round the clock access to a 'mini lecture' enabling them to use the feedback at a time and place of their choosing. We have experimented with screencast feedback in a number of chemistry modules, focussing on feedback for in-class tests and problem-solving activities.

In this communication we will look at screencasting as a means of providing efficient and effective whole class feedback, highlighting the strengths and challenges of this technology. We will discuss the experiences of both students who receive screencast feedback and tutors who provide it, and finish with our suggestions for best practice in the area.

\section{Introduction}

Feedback on assessed work may be defined as information provided by a teacher regarding aspects of performance or understanding and should show students how to bridge the gap between their level of performance or understanding and the level required to meet the intended learning outcomes (adapted from Hattie ${ }^{1}$ and Sadler ${ }^{2}$ contained within reference 1). Effective feedback from a student perspective must be timely and in a format that students can use in revision or in future assignments. Recent National Student Survey results have indicated that students are less satisfied with the speed of marking and feedback (Question 7: Feedback on my work has been prompt) and the ability of feedback to aid understanding (Question 9: Feedback on my work has helped me clarify things I did not understand $)^{3}$. For staff, providing detailed feedback is often a trade-off between speed of marking, and level of detail required. It is also a source of some frustration when students continue to make the same errors despite apparently receiving detailed feedback on previous, related assignments.

Encouraging students to reflect upon feedback has been identified as the third step in assessment and feedback by Sergeant ${ }^{4}$. The first step, assessing the performance, can largely be separated from the second step, providing feedback. This may be useful for students who are unwilling to confront their grade, allowing them to engage in reflective use of feedback. By providing feedback on in-class assessment in the form of model answers with general comments, students may be more likely to incorporate that feedback in their revision process and therefore benefit more from it. Such feedback is also detached from assessment of performance, focussing on the level of understanding required more generally.

Chemistry and Medicinal Chemistry at Keele University has recently seen significant growth in undergraduate student numbers with first year class sizes increasing from 60 in 2008/09 to 100 in 2010/11. The challenges of providing detailed feedback promptly to students has increased proportionately with increasing student numbers, however a 
number of areas in which the feedback process can streamlined somewhat have been identified. These problems are similar to those faced by teachers with little scheduled contact time and when online instruction is indicated. Providing individual feedback on work is a time-consuming and often repetitive process, but a very necessary process. Providing whole class feedback is an opportunity to address problems more generally, particularly those that some

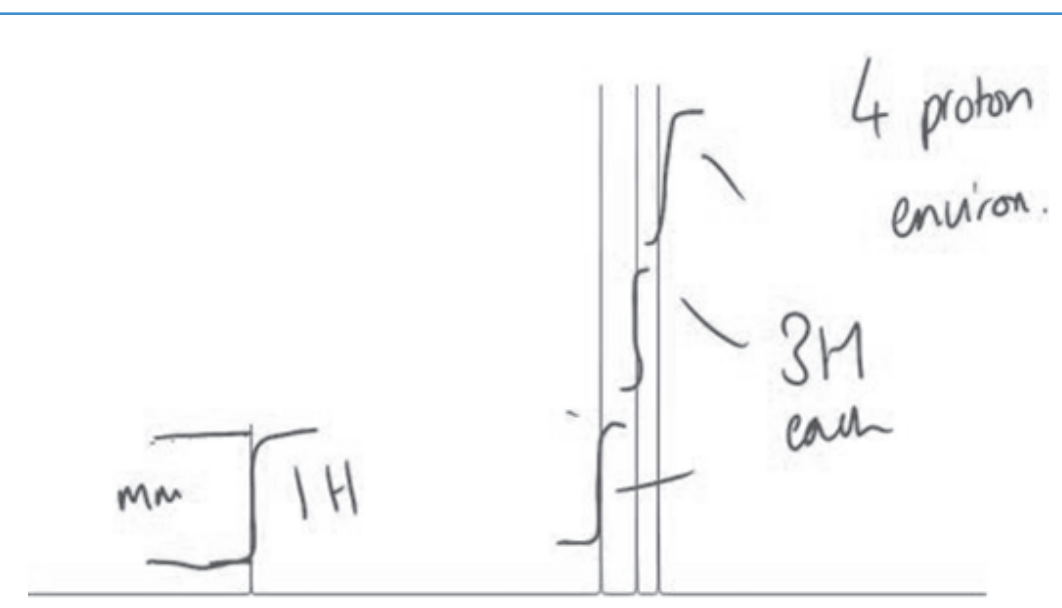

students have not yet made. Feedback can be prepared and released very rapidly after an assessment, even before grades are returned to the students.

For many assignments such as class tests, model answers are often requested by students in addition to a grade and some individual feedback on the script. There are several advantages to providing these:

- Many of our students learn by doing and having a full set of answers to assess their attempts against is beneficial. Students have stated that they revisit and practice class test and problems class questions when revising for end of module exams.

- Students who have not engaged fully with the material at the time of the class tests may not know sufficient to make certain mistakes. For example, a student who has barely attempted to answer a question is unlikely to have made a large number of mistakes, and therefore will not benefit from feedback written on the script unless it provides the full, worked answer. Providing individual feedback on work submitted does not anticipate potential growth in their understanding that comes with revision and practice, and is therefore of limited longer term value.

We have provided typed or handwritten model answers for most class test questions in the past and this has been viewed as useful and adequate by our students. These model answers have often included more general key points of feedback such as 'remember to show all working' or 'UNITS!' An alternative approach is to use some teaching sessions to review assignments and to go through answers with the whole class, allowing greater interactivity. This is not always possible within the constraints of timetables, and may be off-putting for students who have performed well, leading to a lack of engagement.

Screencasting, also known as vodcasting, offers a means of providing mini-lectures working through answers to assessments that students can view at a time and place of their choosing, rather than being forced to listen in a timetabled class. Screencasts are a useful form of e-teaching, and have been successfully used in distance or online

PC running Windows 7 and Camtasia Studio $7^{6}$. Care must be taken when selecting a tablet PC to avoid modern touch screen portable tablets that do not have the processing power necessary to run presentation and screen capture software. Many modern tablets do not come with stylus pens suitable for written annotations, being largely designed for touch screen operation. Screencasts may be recorded using free websites such as Screen $\mathrm{R}^{7}$ or $\mathrm{Jing}^{8}$ but are limited in length and editing facilities. The resulting screencasts are made available on websites. Audio must be recorded directly onto the computer when using these sites and a headset microphone may be useful to improve audio quality.

For screencasts recorded with Camtasia, a digital voice recorder was used in place of the internal microphone to record high quality audio which was then edited into the screencast. The screencasts were edited before production of the video to remove any awkward pauses or stumbles. This enabled the screencasts to be recorded in one attempt. Custom production settings were used within Camatasia Studio 7 to enable a table of contents to be produced in the finished video. This allows students to navigate to a specific part of the screencast, for example a specific PowerPoint slide title, enabling them to revisit content easily. Screencasts were approximately 5 minutes in length (the maximum length for online production) and took around 20 minutes to prepare, record and edit each after the initial learning curve (Fig. 1).

\section{Examples of Use and Evaluation}

\section{First Year Chemistry}

Screencasts were used to provide model answers to a number of class tests in the 2010/11 academic year including those involving spectroscopic data interpretation and main group inorganic chemistry. For first year Chemistry, screencast model answers and feedback were provided in addition to marked class test scripts, but the level of feedback offered on the marked scripts was reduced to only the key points and uncommon errors, and students were directed to consult the screencast model answer feedback. 


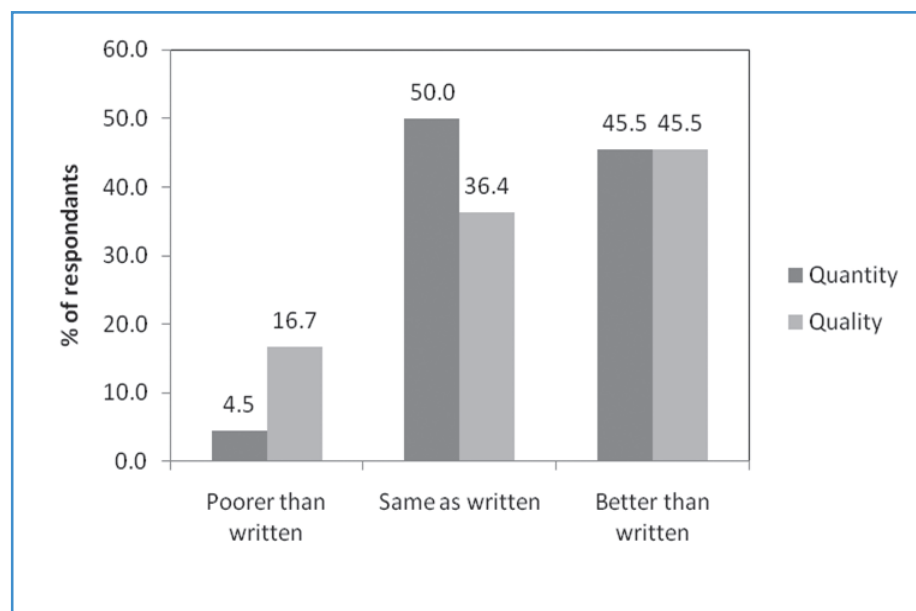

Figure 2: Question: think about screencasts as a way of providing feedback and model answers for class tests in first year modules. How does screencast feedback compare to written in terms of quality and quantity of feedback? Graph gives values as \% of responses, 66 responses received from class of 90 students

To prepare the screencast feedback, PowerPoint slides were created with one question per slide with sufficient room for writing the answers, and a script was prepared by writing out the answers in full on a printout of each slide. An individual screencast was produced for each question to allow students to find the part they required more rapidly, and to reduce the demands on the working memory of the students ${ }^{9}$. Student feedback indicates that this should have been further broken down, perhaps into subsections of questions.

First year chemistry students were asked to evaluate the quantity and quality of whole class screencast feedback as compared to written feedback in a questionnaire about teaching methods conducted in March 2011.

The majority of first year students felt the screencast was equal to, or better than, written feedback in quality and quantity (Fig 2). Many students indicated that they would prefer written feedback as they could identify the specific area of interest more rapidly. Searching through a screencast for a particular section was identified as particularly frustrating; even from students who felt that screencasts on the whole were better than written feedback. While a table of contents, or recording short screencasts for each question can partly work around this concern, a copy of the annotated slides produced during recording can be provided. A text based version can be provided for students with specific needs if required.

"I found the video feedback to be very helpful. It allowed me to look at my answers in the comfort of my own home, without using up valuable problem class / lecture time. [The] commentary highlighted some points that would not have been easily conveyed in writing alone." - First Year Student

While the screencasts are better than written, they are also longer and therefore it takes more time to review a single question. Separate screencasts for each question could make it easier to sort through." - First Year Student
Third Year Chemistry

A recent development in assessment in $3^{\text {rd }}$ year chemistry at Keele is the setting of test questions based on research papers, requiring students to locate, extract and analyse/ interpret complex data and information. The examples for which screencast feedback was provided were based on advanced kinetics, activation parameters and inorganic/ organic reaction mechanisms. Although test questions are seen in advance, students did not know which would be selected for the summative test. Copies of the research articles were provided to the students during the test. This type of assessment was ideally suited to screencast feedback because it enabled the problem-solving process and thought processes of the tutor to be communicated in a manner that would be very difficult with static written feedback. The sequential introduction of material with forward and back referencing and supported by audio to emphasise key points and common errors adds additional dimensions to the feedback. The screencast feedback for these test questions was recorded, edited and produced using Camtasia on a standard laptop with in-built microphone, as an alternative to the tablet PC. PowerPoint slides were prepared in advance using animation to make parts of the answers appear as required. The class viewed this very positively resulting in a number (in 11 out of 25 questionnaires) of unprompted comments on the screencasts in end of module evaluations.

"This is a great tool to use when going back over questions, if I was just provided with the equations without the audio I wouldn't understand your thinking and also some of the basic maths (I don't think I could apply what I would see to another example )." -Third Year Student

\section{Tutor Experiences}

Production of screencast feedback was more time consuming than simply providing written or typed model answers to the class, but student responses indicates that this is time well spent. Incorporating generic comments into the screencast such as indicating the most common errors and how to avoid them was extremely useful, giving a level of detail in the feedback that could not be achieved on individual scripts.

\section{Summary and Suggestions for Best Practice}

Our experiences of providing screencast feedback on problem solving questions to whole classes have been largely successful, and well received by the students. While there is an initial learning curve with this technology, the process can be extremely efficient. We have some suggestions based on our experience that may help anyone considering this method of feedback.

- Consider the length of each screencast carefully and break larger questions into sections.

- Stay focussed on the topic of the screencast and avoid incorporating additional examples or too many alternative explanations.

- Organise material logically to minimise demands on working memory.

- Write a script or plan for each screencast and follow it.

- Ensure that students have suitable access to computer facilities with sound and sufficient IT skills to access the screencasts. 
- Ensure your audio is of sufficient quality - inbuilt microphones may lead to quiet recordings.

- Turn off email or other programmes - pop up notifications will be recorded on screencasts!

\section{Acknowledgements}

The authors are grateful to the JISC funded project STAF (Supporting staff in the use of Technology for Assessing and giving Feedback) for providing a copy of Camtasia for use in feedback. The authors would like to thank $1^{\text {st }}$ and $3^{\text {rd }}$ year (2010-11) Keele University Chemistry/Medicinal Chemistry undergraduate students for their valuable feedback.

\section{References}

1. Hattie, J., and Timperley, H. (2007) The Power of Feedback, Review of Educational Research, 77 (1) 81-112.

2. Sadler, R. (1989) Formative Assessment and the Design of Instructional Systems, Instructional Science, 18 119-144 as described in Hattie ${ }^{1}$

3. NSS 2010 <www.hefce.ac.uk/learning/nss/data/2010/ > and associated excel sheet. Accessed 20/04/10

4. Sargeant, J.M., Mann, K. V., van der Vleuten, C.P., and Metsemakers, J.F. (2009) Reflection: A Link Between Receiving and Using Assessment Feedback, Advances in Health Science Education, 14 399-410

5. Falconer, J.L., deGrazia, J., Medlin, J. W., and Holmberg, M. P. (2009) Using Screencasts in CHE Courses, Chemical Engineering Education, 34 (4) 302 - 305

6. Camtasia Studio <www.techsmith.com/camtasia/> Accessed 13/05/11

7. ScreenR <www.screenr.com/> Accessed 13/05/11

8. Jing $<$ www.techsmith.com/jing/> Accessed 13/05/11

9. Oud, J. (2009) Guidelines for Effective Online Instruction Using Multimedia Screencasts, Reference Services Review, 37 (2) 164-177

10. Project STAF $<$ www.jisc.ac.uk/whatwedo/programmes/bcap/ keele.aspx> Accessed 13/05/11 